\title{
False positive and false negative diagnoses of prostate cancer at multi-parametric prostate MRI in active surveillance
}

\author{
Jeffrey S. Quon ${ }^{1} \cdot$ Bardia Moosavi $^{1} \cdot$ Maneesh Khanna $^{2}$ - Trevor A. Flood ${ }^{3}$. \\ Christopher S. Lim ${ }^{1} \cdot$ Nicola Schieda ${ }^{1}$
}

Received: 7 January 2015 /Revised: 2 May 2015 / Accepted: 8 May 2015 / Published online: 23 May 2015

(C) The Author(s) 2015. This article is published with open access at Springerlink.com

\begin{abstract}
MP-MRI is a critical component in active surveillance (AS) of prostate cancer $(\mathrm{PCa})$ because of a high negative predictive value for clinically significant tumours. This review illustrates pitfalls of MP-MRI and how to recognise and avoid them. The anterior fibromuscular stroma and central zone are low signal on T2W-MRI/apparent diffusion coefficient (ADC), resembling PCa. Location, progressive enhancement and low signal on $b \geq 1000 \mathrm{~mm}^{2} / \mathrm{s}$ echo-planar images (EPI) are differentiating features. BPH can mimic PCa. Glandular BPH shows increased T2W/ADC signal, cystic change and progressive enhancement; however, stromal $\mathrm{BPH}$ resembles transition zone (TZ) PCa. A rounded morphology, low T2 signal capsule and posterior/superior location favour stromal BPH. Acute/
\end{abstract}

Nicola Schieda

nschieda@toh.on.ca

Jeffrey S. Quon

jquon@toh.on.ca

Bardia Moosavi

bmoosavi@toh.on.ca

Maneesh Khanna

khanna.maneesh@gmail.com

Trevor A. Flood

tflood@toh.on.ca

Christopher S. Lim

clim@toh.on.ca

1 Department of Medical Imaging, The Ottawa Hospital, The University of Ottawa, 1053 Carling Avenue, Ottawa, ON, Canada K1Y 4E9

2 Department of Radiology, Hamad General Hospital, Doha, Qatar

3 Department of Pathology, The Ottawa Hospital, The University of Ottawa, 501 Smyth Road, Ottawa, ON, Canada K1H 8 L6 chronic prostatitis mimics PCa at MP-MRI, with differentiation mainly on clinical grounds. Visual analysis of diffusion-weighted MRI must include EPI and appropriate windowing of ADC. Quantitative ADC analysis is limited by lack of standardization; the ADC ratio and ADC histogram analysis are alternatives to mean values. DCE lacks standardisation and has limited utility in the TZ, where T2W/DWI are favoured. Targeted TRUS-guided biopsies of MR-detected lesions are challenging. Lesions detected on MP-MRI may not be perfectly targeted with TRUS and this must be considered when faced with a suspicious lesion on MP-MRI and a negative targeted TRUS biopsy histopathological result.

\section{Keypoints}

- Multi-parametric MRI plays a critical role in prostate cancer active surveillance.

- Low T2W signal intensity structures appear dark on ADC, potentially simulating cancer.

- Stromal BPH mimics cancer at DWI and DCE.

- Long b value trace EPI should be reviewed

- Targeted biopsy of MR-detected lesions using TRUS guidance may be challenging.

Keywords Prostate cancer - Active surveillance of prostate cancer · Multi-parametric MRI · Targeted biopsy ·

Diffusion-weighted imaging

\section{Introduction}

Prostate cancer $(\mathrm{PCa})$ is the most common cancer among males in North America [1, 2] and the most common solid neoplasm in Europe [3]. PCa is typically diagnosed with non-targeted systematic trans-rectal ultrasound (TRUS)-guided biopsy in 
patients with an elevated prostate serum antigen (PSA) level and/or abnormal digital rectal examination (DRE). The management of a particular patient with PCa is multi-factorial and patient specific due to the biological heterogeneity of $\mathrm{PCa}$. Traditional treatment of $\mathrm{PCa}$ varies from radical prostatectomy (RP) or radiotherapy (RT) to watchful waiting (delayed symptomatic non-curative treatment of apparently localised PCa in males who are not candidates for aggressive local therapy) [1, 3]. Active surveillance (AS) is defined as the expectant management (deferred immediate therapy) of PCa in carefully selected males with localised disease considered to be at low risk for progression [4]. AS differs from watchful waiting because definitive treatment is used in patients managed with AS when there is evidence that the patient is at an increased risk for disease progression $[4,5]$. AS has become the treatment of choice for low-grade, low-volume tumours $[6,7]$ and is heavily reliant on accurate detection of tumour, accurate estimate of tumour volume and accurate Gleason grading of tumour [6, 7].

Non-targeted TRUS-guided biopsy typically obtains 6-12 core biopsies of the peripheral zone (PZ), which harbours approximately $70 \%$ of cancers $[1,8]$. The limitations of nontargeted TRUS-guided biopsy are well known [8] with an estimated $20 \%$ false-negative rate [9-11]. Furthermore, nontargeted TRUS-guided biopsy may yield unreliable information regarding the volume, extent and aggressiveness of $\mathrm{PCa}$; it is has been reported that up to $30-45 \%$ of patients are upgraded/ upstaged from their initial diagnosis at TRUS-guided needle biopsy after RP [12]. Moreover, certain areas of the prostate gland [i.e., the anterior gland, transition zone (TZ) and apex] are known to be under-sampled or not sampled at all at routine nontargeted TRUS-guided biopsy and are now increasingly being recognised as areas that may contain clinically significant (CS) tumours [13]. These limitations are of critical importance in AS, where treatment decisions are based on risk stratification and dependent on accurate Gleason grading of tumours [4].

Multi-parametric (MP) MRI [diffusion-weighted imaging (DWI)+dynamic contrast enhancement (DCE) and/or MR spectroscopy] has become the reference standard for prostate imaging endorsed by both the American College of Radiology (ACR) and European Society of Uroradiology (ESUR) [14, 15] (Table 1). Two recent meta-analyses concluded that MP-MRI has a high negative predictive value for the detection of CS cancers $[16,17]$, and it has been shown previously that MPMRI can estimate grade of PCa compared to histopathology results with a reasonable degree of accuracy [18]. Due to the ability of MP-MRI to detect clinically significant (higher volume Gleason score $\geq 7$ ) tumours with high degrees of accuracy, it has become of tremendous value in AS [19]. Recent studies have demonstrated that MP-MRI can help to determine eligibility for AS [20] and potentially reclassify patients already enrolled in AS before repeat biopsies [21]. In the UK, the National Institute for Health and Care Excellence (NICE) guidelines currently mandate MP-MRI be performed at the onset of AS protocols and that

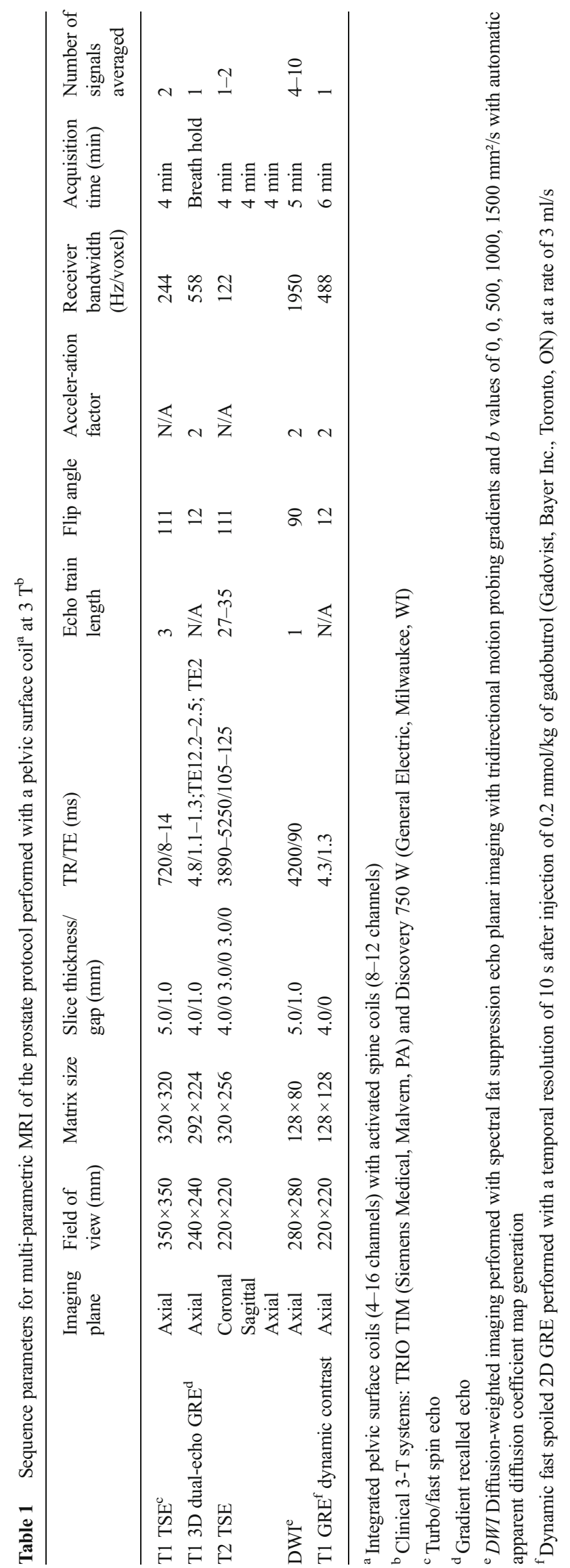


MP-MRI be performed in patients while they are enrolled in AS when there is concern about clinical or PSA changes [22].

A variety of interpretive and technical pitfalls may be encountered at MP-MRI of the prostate. A failure to recognise and correct these errors in AS patients can result in suboptimal care. False-positive diagnoses of areas of potential cancers at MP-MRI create clinical uncertainty and often lead to multiple unnecessary biopsies or in certain cases surgical management of low-grade, low-volume disease. Moreover, a failure to recognise clinically significant cancers in males being considered for or treated with AS could result in suboptimal patient outcomes. The purpose of this review is to illustrate both interpretive and technical pitfalls encountered at MP-MRI in the active surveillance population and how to detect, correct and avoid them.

\section{Interpretive pitfalls}

\section{(1) Normal anatomic structures can mimic anterior and TZ cancers}

A detailed understanding of the normal zonal anatomy of the prostate is essential for interpretation of prostate MRI. In 1981, McNeal [23] described the three distinct prostate zonal regions: (1) the peripheral zone, (2) transition zone and (3) central zone $(\mathrm{CZ})$. The prostatic zonal anatomy is best depicted at T2-weighted (W) MRI (Fig. 1). The PZ is hyperintense on $\mathrm{T} 2 \mathrm{~W}$ because of abundant glandular tissue, is located at the periphery of the gland and harbours $70 \%$ of $\mathrm{PCa}$ [24] (Fig. 1). Previously, it was thought that the TZ [the site of benign prostatic hyperplasia (BPH)] and the $\mathrm{CZ}$ (which surrounds the ejaculatory ducts, is located mainly at the base of the prostate, is posterior to the $\mathrm{TZ}$ and the urethra, and is proximal to the verumontanum) could not be differentiated at imaging and these areas were collectively referred to as the central gland $[25,26]$. The central gland in most adult males consists of hypertrophied $\mathrm{TZ}$ that compresses the $\mathrm{CZ}$ against the surgical capsule [25]. Currently, it is acknowledged that the $\mathrm{CZ}$ can be identified separately from the TZ in up to 4/5 of males and the CZ appears as a symmetric band of homogeneously low signal intensity (SI) on T2W MRI and apparent diffusion coefficient (ADC) maps best seen at the prostate base [25], (Fig. 1).

Accurate identification of the $\mathrm{CZ}$ is critical as, in our experience, many false-positive interpretations of $\mathrm{PCa}$ occur when mistaking the $\mathrm{CZ}$ for $\mathrm{PCa}$. The $\mathrm{CZ}$ can be diagnosed by noting its symmetry (particularly on coronal T2W images) and typical location adjacent to the ejaculatory ducts [25, 26]. Functional imaging techniques also aid in the differentiation of $\mathrm{CZ}$
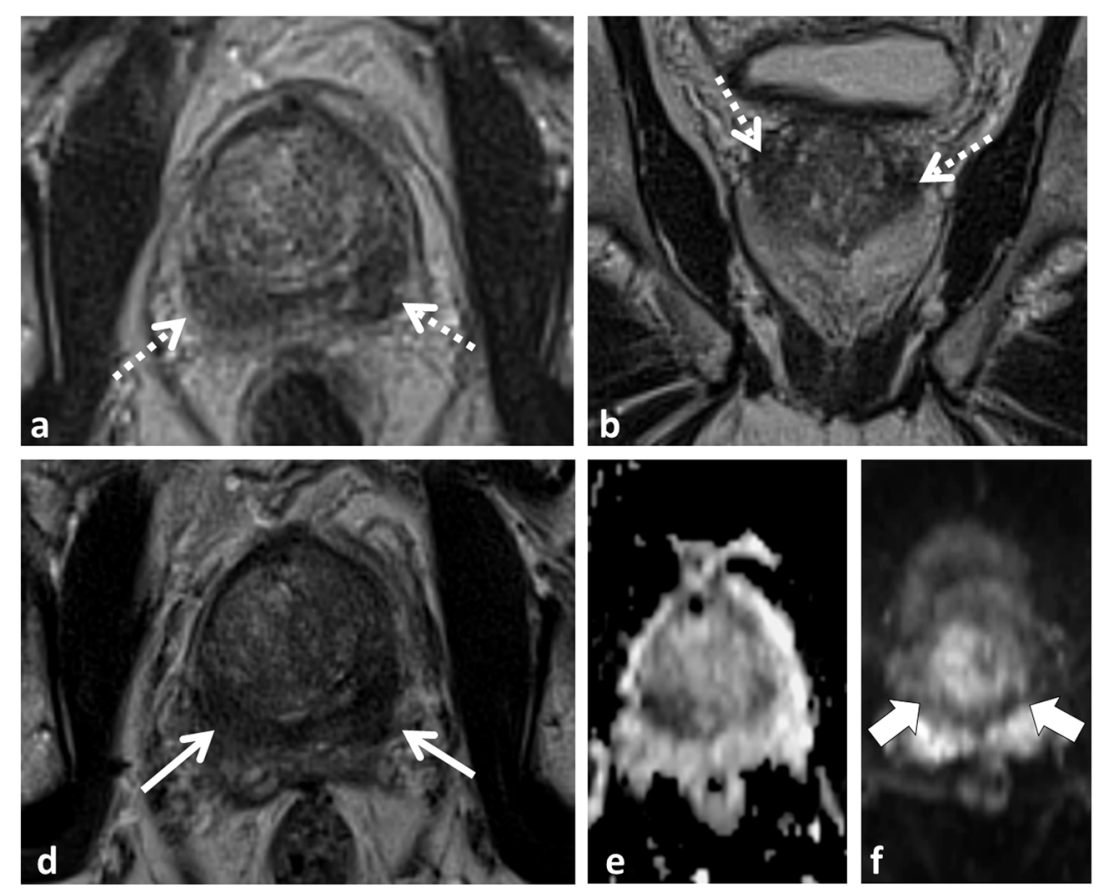
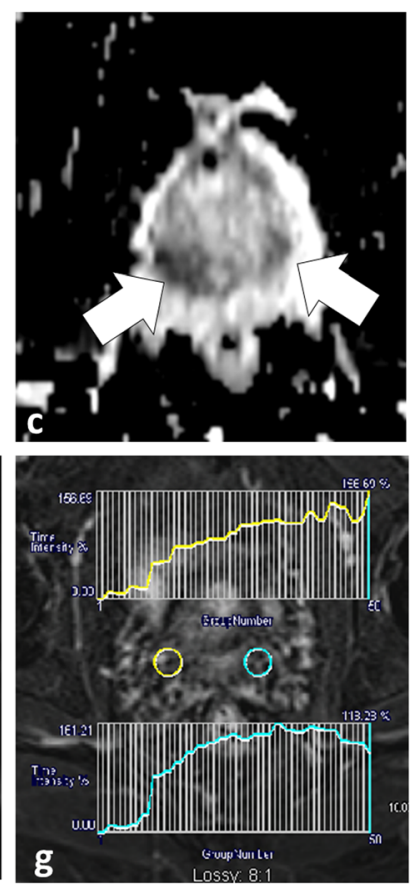

Fig. 1 A 54-year-old male with low volume Gleason score 3+3=6 PCa at TRUS-guided biopsy in the right middle peripheral zone $(P Z)$. MPMRI was performed because of rising PSA to exclude clinically significant $(C S)$ tumour. Axial (a) and coronal (b) T2-weighted (T2W) turbo spin echo (TSE) images demonstrate bilateral foci of low T2 signal intensity (SI) at the prostate base (dotted arrows) adjacent to the insertion of the vas deferens (not shown). Axial apparent diffusion coefficient (ADC) map (c) at the same level demonstrates corresponding low SI (white arrows). These areas were described as suspicious and repeat targeted TRUS-guided biopsy was suggested. A repeat biopsy with multiple cores through both areas yielded only normal prostatic tissue. A repeat MRI performed 2 years later demonstrates similar findings on the T2W (d) and ADC map (e), which represent the normal central zone. Corresponding b1000 $\mathrm{mm}^{2} / \mathrm{s}$ trace echo-planar image (EPI) does not show concordant areas of increased SI (white arrows in $\mathbf{f}$ ) and there is benign progressive enhancement on dynamic contrast-enhanced (DCE) imaging (g) 
from $\mathrm{PCa}$. In the normal $\mathrm{CZ}$, low $\mathrm{SI}$ on $\mathrm{ADC}$ is due to inherently low T2 SI not true restricted diffusion ("T2 black hole effect"); see Technical Pitfall 2. In the normal CZ, there is no true diffusion restriction; therefore, there should be no increased SI on long ( $\left.b \geq 1000 \mathrm{~mm}^{2} / \mathrm{s}\right)$ EPI (Figs. 1 and 2$)$. The enhancement pattern of the $\mathrm{CZ}$ at DCE has not, to our knowledge, been described; however, in our experience, a progressive type 1 enhancement is typical of the CZ (Fig. 1). CZ PCas are aggressive [26-28], but account for less than $5 \%$ of $\mathrm{PCa}$ [26]. An asymmetry of the central zone should raise suspicion for a possible tumour [25]; however, it is more common to observe a slight asymmetry in the thickness of the CZ because of the orientation of the prostate gland and the plane of imaging (Fig. 2). To diagnose the rare CZ PCa, asymmetry at $\mathrm{T} 2 \mathrm{~W}$ should be confirmed in multiple planes and corresponding functional imaging findings such as increased signal intensity on long $b$ value trace EPIs and/or suspicious contrast kinetics at DCE are helpful, as these latter findings are not expected in the normal CZ (Fig. 2). Ultimately targeted biopsy or MRI follow-up may be necessary for suspicious areas in the CZ.

The anterior fibromuscular stroma (AFMS) is a normal structure at the extreme anterior midline of the prostate gland, which is inseparable from the surrounding normal prostatic stroma $[25,29]$. The AFMS contains no glandular tissue and is composed of widely spaced smooth muscle cells. It appears homogeneously hypointense on T2W MRI and because of this inherent low T2W SI will appear as low SI on ADC maps. Differentiation of the AFMS from anterior cancer is established by noting the midline location, well-defined margins, lack of true diffusion restriction (absence of high SI on $\mathrm{b} \geq 1000 \mathrm{~mm}^{2} / \mathrm{s}$ EPI) and a benign progressive type 1 enhancement pattern at DCE (Fig. 3) [25, 30]. PCa arising in the extreme anterior prostate can resemble the AFMS and in many cases anterior tumors can extend up to or invade the AFMS. Both lesions are low SI on T2W; however, anterior PCa is more lenticular or polygonal in shape, has ill-defined or smudged borders, and the bulk of the lesion tends to be off midline (Fig. 4) [31]. The use of functional imaging sequences is contributory (Fig. 4) [30]. DWI increases accuracy for detection of anterior cancers because anterior tumours will be of low SI on ADC because of true restricted diffusion, demonstrating increased SI on trace $b \geq 1000 \mathrm{~mm}^{2} / \mathrm{s}$ EPI [32]. The utility of DCE in the TZ and anterior prostate is controversial [33]; however, authors have demonstrated more aggressive enhancement patterns in anterior PCa [34-37]. In our experience, DCE can be beneficial to discriminate anterior $\mathrm{PCa}$ from AFMS; however, overlap with stromal BPH limits its utility in the TZ (discussed later) [35].

\section{(2) Post-biopsy haemorrhage can mimic PZ PCa on T2W MRI}

Detection of $\mathrm{PCa}$ on $\mathrm{T} 2 \mathrm{~W}$ is predicated on the ability to discriminate low T2 SI tumour from the normal adjacent high T2 SI PZ [15, 38-40]. The glandular tissue of the PZ contains high concentrations of citrate, which is a natural anticoagulant; therefore, post-procedural haemorrhage is commonly observed at MRI after prostate biopsies and can persist for up to 4 months after the procedure [41]. Areas of postbiopsy haemorrhage are characteristically hypointense on T2W MRI in up to $80 \%$ of cases and can mimic PCa [41] (Fig. 5). This pitfall is easily avoided by cross-referencing $\mathrm{T} 2 \mathrm{~W}$ to pre-contrast $\mathrm{T} 1 \mathrm{~W}$ imaging because areas of haemorrhage will also be characteristically hyperintense on $\mathrm{T} 1 \mathrm{~W}$ (Fig. 5).

Many institutions continue to defer prostate MRI studies until 3-8 weeks have passed after the biopsy to reduce the amount of post-biopsy haemorrhage and facilitate interpretation. With the advent of functional imaging sequences postbiopsy haemorrhage is, in our experience, much less problematic. Areas of tumour have been shown to be reliably differentiated from haemorrhage using DWI, and DCE analysis can be interpreted through image subtraction [42] (Fig. 5). Moreover, because PCa contains less citrate than the normal PZ,
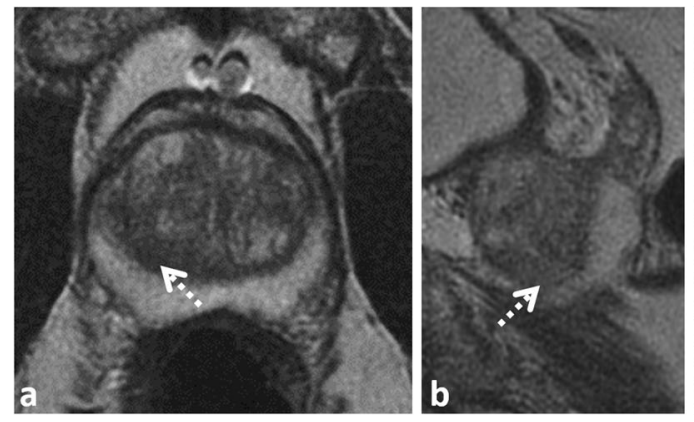

Fig. 2 A 51-year-old patient with low-volume Gleason score 3+3=6 at non-targeted TRUS-guided biopsy. MP-MRI was performed at onset of active surveillance (AS) to exclude higher grade tumour. Axial (a) and sagittal (b) T2W images demonstrate an ill-defined ovoid lesion of low T2 SI at the junction of the right PZ and transition zone (TZ) (dotted white arrow) along the course of the surgical capsule. Axial ADC map image
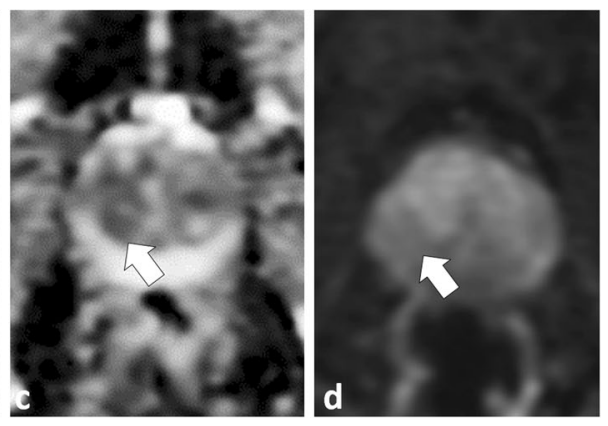

(c) at the same level demonstrates corresponding low SI (white arrow). This area was considered suspicious for tumour. Targeted TRUS biopsy was performed and yielded only normal prostate tissue. The area did not change on follow-up MP-MRI and represents asymmetry of the normal central zone. In retrospect, corresponding b1000 $\mathrm{mm}^{2} / \mathrm{s}$ trace EPI (d) did not show increased signal (white arrow) in this area 

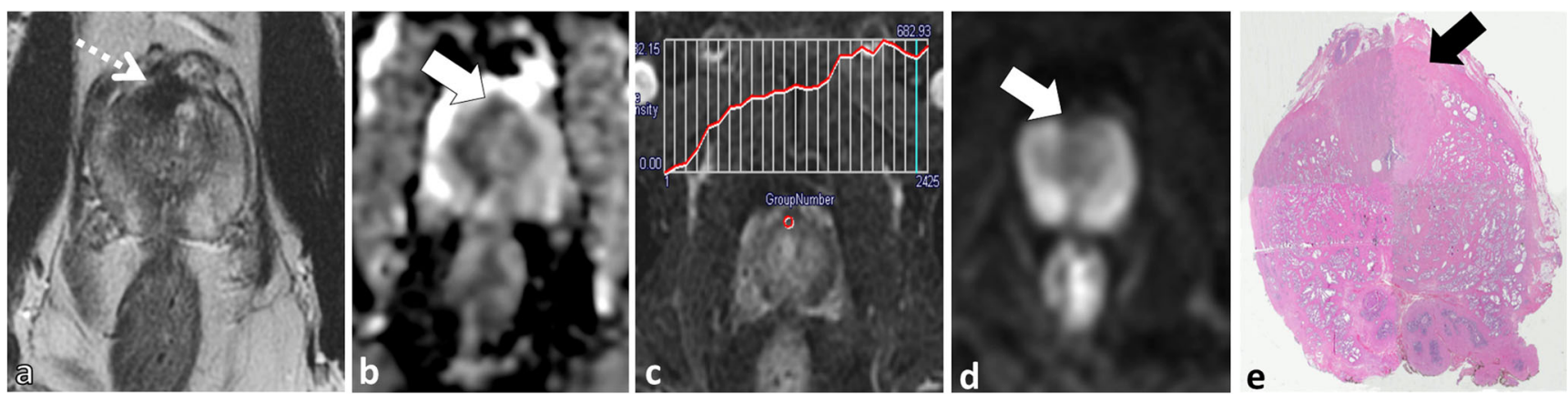

Fig. 3 A 54-year-old patient with elevated and rising PSA. Axial T2W TSE (a) demonstrates a low SI focus in the anterior midline (dotted arrow) with corresponding low SI (white arrow) on the axial ADC map (b). This area was reported as suspicious for tumour. Repeat non-targeted biopsies of the $\mathrm{PZ}$ revealed Gleason score $3+4=7$ tumour in the right PZ (not shown) and the patient underwent radical prostatectomy (RP). The structure highlighted on MRI is the normal anterior fibromuscular stroma

areas that are devoid of haemorrhage in the PZ after biopsy may actually highlight the tumour on T1W imaging ("haemorrhage exclusion sign") [42]. This imaging finding has recently been shown to have $>95 \%$ positive predictive value for tumour localisation when combined with characteristic imaging findings at T2W MRI [42].

\section{(3) Benign prostatic hyperplasia $(\mathrm{BPH})$ resembles $\mathbf{T Z}$ PCa}

$\mathrm{BPH}$ is extremely common in the $\mathrm{TZ}$ and its prevalence increases with age. BPH can be nodular and TZ nodules are commonly encountered at MP-MRI [35], and nodular BPH may mimic TZ PCa [25]. Nodular BPH is categorised into three main subtypes: glandular, stromal and mixed.

Glandular BPH is characterised histologically by hyperplasia of glandular tissue with papillary buds, infoldings and cysts. Glandular BPH is readily differentiated from $\mathrm{TZ} \mathrm{PCa}$ at MP-MRI [35] and typical features include a wellcircumscribed, sharply demarcated and rounded shape, a
(AFMS); note: characteristic midline anterior location on axial T2 (a) and benign progressive enhancement on DCE (c). Also note that low SI on the ADC (b) map is due to inherently low T2 SI rather than restricted diffusion; there is a lack of increased SI on trace b1000 $\mathrm{mm}^{2} / \mathrm{s}$ EPI (d). Whole-mount histopathology image of the RP specimen (e) demonstrates the AFMS (black arrow), which is predominantly composed of smooth muscle that blends with the overlying prostate stroma continuous low T2W SI rim, increased T2W SI often with cystic change, low SI on long $\geq 1000 \mathrm{~mm}^{2} / \mathrm{s} b$ value EPI, "T2 shine-through" on ADC maps and benign/progressive/type I contrast kinetics with a low transfer constant on DCE (Fig. 6) $[25,35]$.

The presence of increased smooth muscle, lymphocytes and ducts (not associated with prostatitis) and reduced elastic tissue characterises stromal BPH at histopathology. Stromal $\mathrm{BPH}$ nodules can be difficult to differentiate from TZ PCa [35] (Figs. 6 and 7). Stromal BPH is typically of low T2W SI, similar to TZ PCa. Stromal BPH is also of low signal intensity on ADC maps because of both inherently low T2W SI and true restricted diffusion (due to compact cellularity) and may or may not have increased SI on long $\mathrm{b} \geq 1000 \mathrm{~mm}^{2} / \mathrm{s}$ trace EPI. Furthermore, ADC values in stromal $\mathrm{BPH}$ are low and overlap with TZ PCa $[25,35]$. DCE is limited to differentiate stromal $\mathrm{BPH}$ from $\mathrm{TZ} \mathrm{PCa}$, because enhancement patterns of stromal BPH overlap with TZ PCa [33-35]. Previous authors have reported lower ADC and higher K-trans values in TZ PCa compared to stromal BPH,
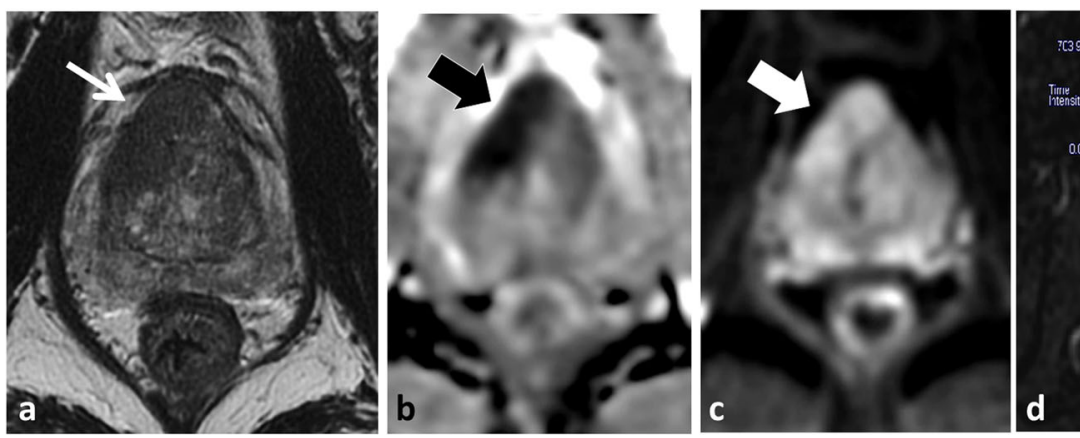

Fig. 4 A 50-year-old patient with rising PSA and a typical anterior tumour involving the right side of the gland. Axial T2W TSE (a) demonstrates an amorphous, off midline, lenticular-shaped, ill-defined low T2 SI region (white arrow) with low SI (black arrow) on the ADC (b) map due to restricted diffusion (note increased SI on trace b1000 $\mathrm{mm}^{2} /$ s EPI (white arrow in c) and type III contrast curve kinetics on DCE (d). A targeted TRUS biopsy was performed and confirmed Gleason score 4+ $3=7$ tumour anteriorly. Corresponding microscopic image from TRUS biopsy (e) demonstrates Gleason pattern 4 tumour 

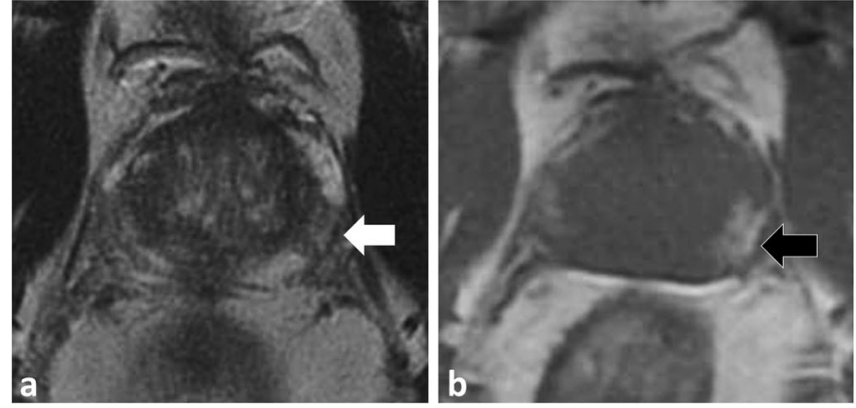

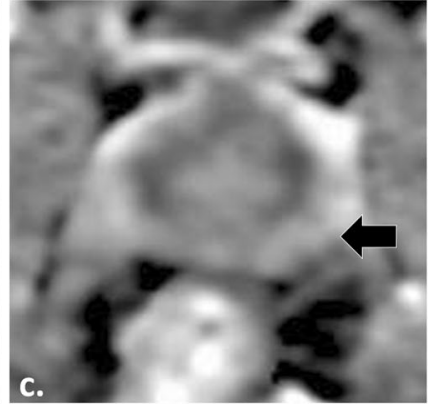

demonstrates increased T1 SI (black arrow) in keeping with postbiopsy haemorrhage. Axial ADC map (c) does not reveal the lesion (black arrow), which was readily diagnosed as post-biopsy haemorrhage

anterior fibromuscular stroma but rarely extend to the posterior PZ [44]. It should be noted that in some instances, when there is uncertainty at MP-MRI regarding the diagnosis of TZ PCa versus stromal BPH due to overlap in imaging features, a targeted biopsy or follow-up MP-MRI may be suggested (Fig. 6).

\section{(4) Acute and chronic prostatitis mimics PCa}

Acute bacterial prostatitis (ABP) is commonly encountered in clinical practice and often managed conservatively without imaging or surgical intervention [38]. When patients with $\mathrm{ABP}$ are imaged in the acute setting, prostatitis can mimic PCa at MP-MRI, demonstrating low SI on T2WI, restricted diffusion [45] and enhancement patterns that overlap with $\mathrm{PCa}$ [25] (Fig. 8). Although PCa has been reported to demonstrate more well-defined borders and nodular appearance compared to prostatitis and lower ADC values [45, 46], aside from the presence of abscess formation in prostatitis, differentiation is mainly based on clinical grounds.
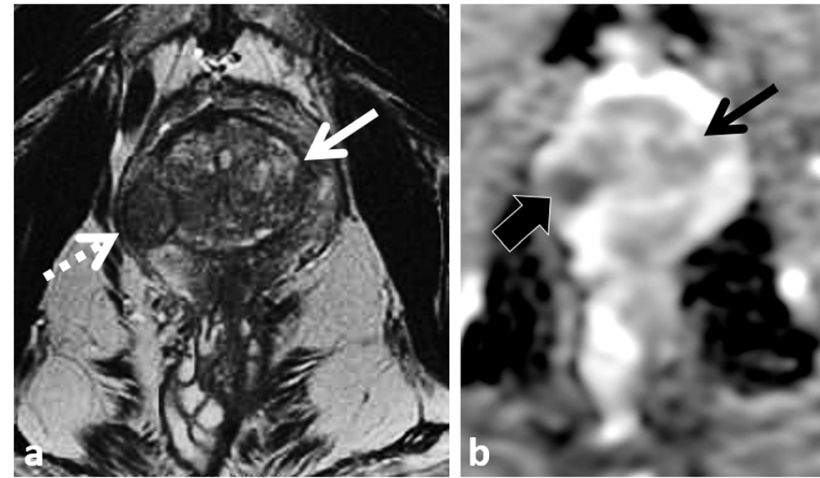

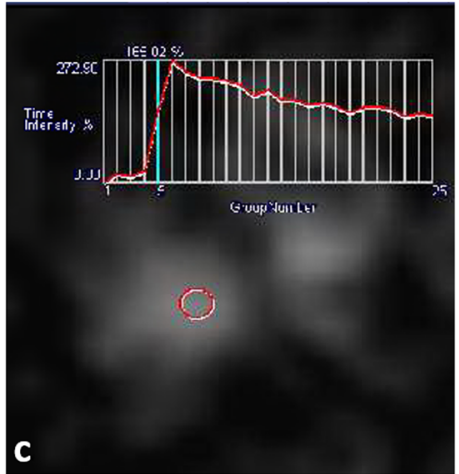

shine-through on the ADC map (black arrow in b) and is characteristic of glandular BPH. The other nodule demonstrates restricted diffusion (thick black arrow in b) and type III kinetics at DCE (c). A diagnosis of prostate cancer was suggested. Targeted biopsy revealed normal prostatic tissue and stromal BPH. Follow-up MRI (not shown) demonstrated no change and the PSA was stable. In retrospect, the nodule is round, wellcircumscribed and demonstrates a complete low T2 SI rim (a), findings that are more in keeping with stromal $\mathrm{BPH}$ rather than cancer 

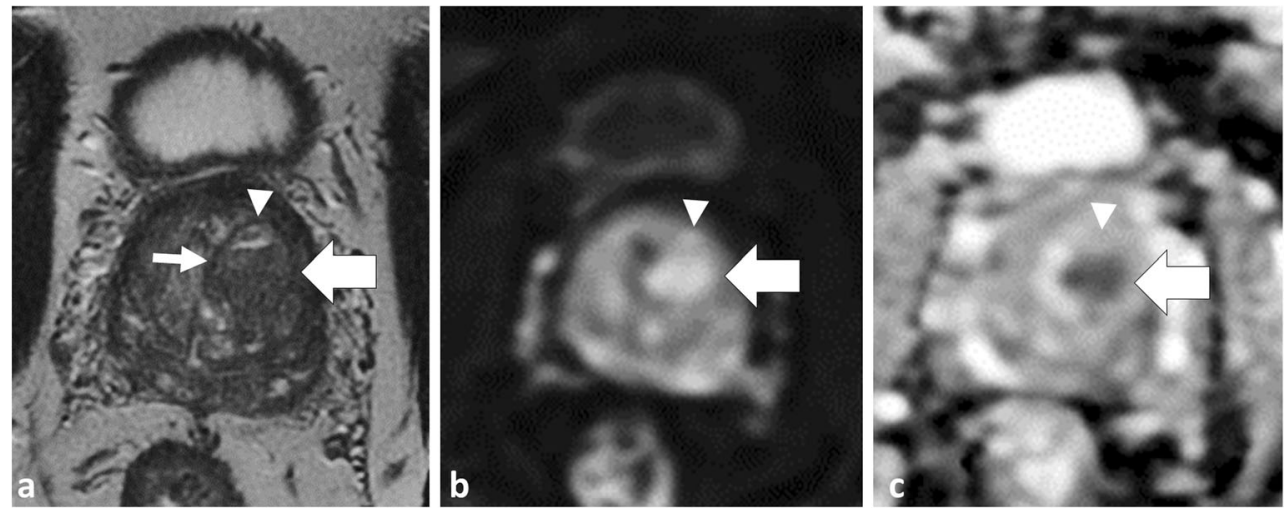

Fig. 7 A 58-year-old patient with low-volume, low Gleason score 3+3= 6 tumour in the right mid-peripheral zone undergoing MRI prior to repeat biopsy because of rising PSA. Axial T2W TSE (a) image demonstrates a low T2 SI nodule in the left TZ (thick white arrow). The nodule is predominantly homogeneously of low T2W SI but demonstrates a small focus of cystic change anteriorly (arrowhead). Note that the

Granulomatous prostatitis (GP) is a benign inflammatory entity that may also be indistinguishable from $\mathrm{PCa}$ at MP-MRI, demonstrating low SI on T2W, restricted diffusion and suspicious enhancement at DCE [47]. Moreover, granulomatous prostatitis can involve the periprostatic fat or seminal vesicles, mimicking extraprostatic spread of PCa (Fig. 9). With the correct clinical history [e.g., previous bacille Calmette-Guerin therapy for bladder cancer, tuberculous prostatitis or nodule is round and demonstrates a continuous low T2W SI rim (thin white arrow). The nodule demonstrates restricted diffusion, increased SI on trace b1000 $\mathrm{mm}^{2} / \mathrm{s}$ EPI (b) and low SI on the ADC map (c) (thick white arrows). Note that cystic change demonstrates T2 shine-through (arrowhead). Imaging findings are characteristic of a mixed but predominantly stromal BPH nodule

previous intervention such as transurethral resection of the prostate (TURP)], granulomatous prostatitis may be favoured over PCa [25]; however, histological confirmation is often required [47].

In the chronic setting, areas of prostatitis can mimic $\mathrm{PCa}$ because they will demonstrate low T2W SI [48]. Functional imaging techniques can be useful to differentiate areas of chronic prostatitis from cancer (Fig. 10) but there is overlap in imaging features $[49,50]$ and targeted biopsy or MP-MRI
Fig. 8 Acute prostatitis in a 46year-old patient with elevated PSA. Axial T2W TSE (a) demonstrates an amorphous area of low T2 SI (dotted arrow) in the left middle PZ with corresponding low SI on ADC map (black arrow in b) due to restricted diffusion, note increased SI on trace b1000 $\mathrm{mm}^{2} /$ $\mathrm{s}$ EPI (white arrow in $\mathbf{c}$ ). There is an indeterminate type II contrast curve (plateau kinetics) on DCE in (d). A differential diagnosis of prostatitis or cancer was provided, and biopsy or follow-up was suggested. The patient had typical findings of acute bacterial prostatitis clinically and was treated with antibiotics with normalisation of PSA post therapy
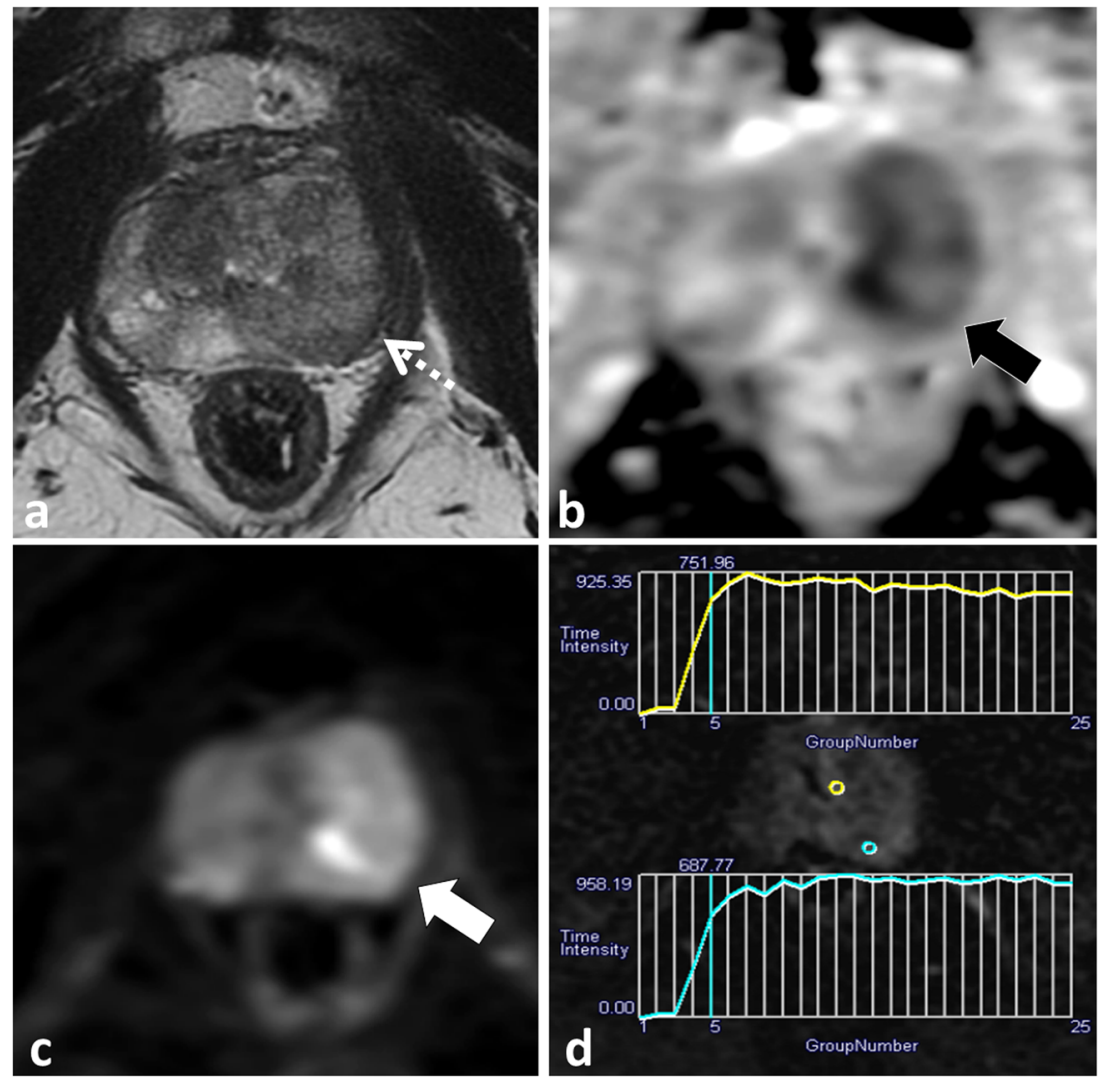


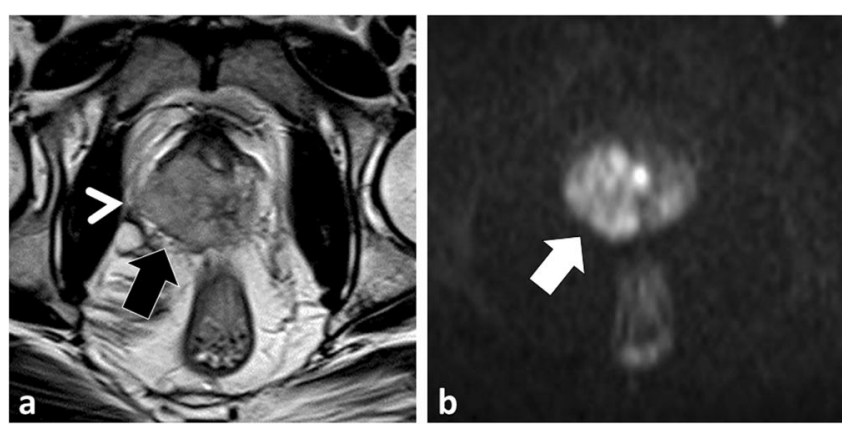

Fig. 9 A 64-year-old patient with rising PSA and history of recurrent urothelial cell carcinoma of the urinary bladder treated with intra-vesical BCG therapy. Axial T2W image (a) demonstrates a large mass infiltrating throughout the right prostate (black arrow) and breaching the prostate capsule laterally (arrowhead) consistent with extra-prostatic spread.

follow-up of the suspicion region may be considered depending on the level of suspicion for tumour [47].

\section{(5) Ductal variant adenocarcinoma may be occult on T2W MRI}

PCa is divided into the most common acinar adenocarcino$\mathrm{ma}$ and less common non-acinar subtypes. Ductal adenocarcinoma (DCa) is an aggressive variant of $\mathrm{PCa}$ and is the most common of the non-acinar subtypes [51] with a reported incidence of $0.5-6 \%$ [52]. DCa is associated with higher rates of
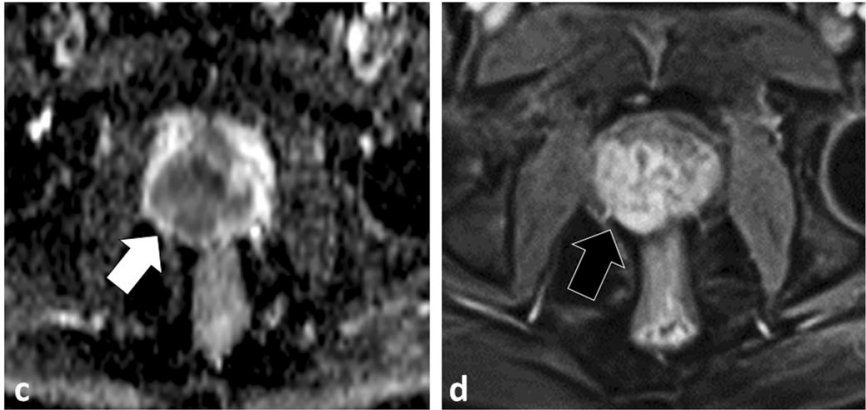

Axial b1000 mm²/s (b) and ADC map (c) demonstrate marked restricted diffusion (white arrows) and axial image from DCE shows marked early mass-like hyper-enhancement (type III curve was depicted and is not shown). TRUS biopsy was performed and histology was compatible with diffuse granulomatous prostatitis

positive surgical margins, extraprostatic extension, vascular invasion, seminal vesicle invasion and metastases [53, 54]. A diagnosis of $\mathrm{DCa}$ at needle biopsy requires definitive therapy and is considered an absolute contraindication for AS [55, 56]. Schieda et al. recently demonstrated that DCa is of increased SI on T2W MRI, which can render the tumour occult [39]; however, the same authors demonstrated that DCa resembles Gleason score $\geq 7$ or higher tumour on DWI and DCE [57]. Therefore, in a lesion with aggressive imaging findings on DWI/DCE and paradoxical increased T2W SI, DCa should be considered [57] (Fig. 11).
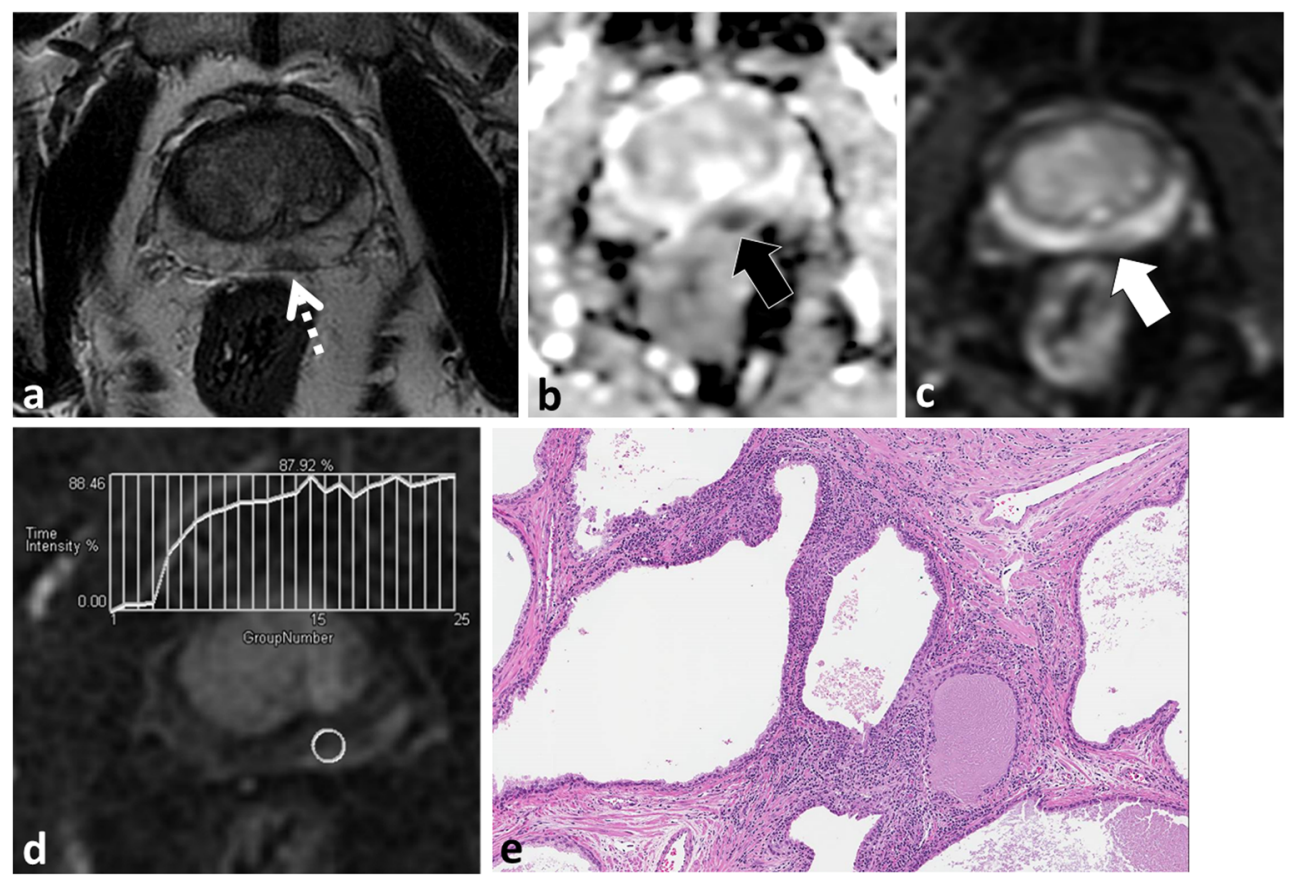

Fig. 10 A 64-year-old patient with Gleason score $3+3=6$ in $<5 \%$ of one core in the left middle PZ underwent MRI prior to enrolment into active surveillance. Axial T2W TSE image (a) demonstrates a low T2 SI focus in the left PZ (dotted arrow) with low SI on ADC (black arrow in b). Based on these findings a diagnosis of potential higher grade tumour was suggested and a repeat biopsy was performed. Saturation biopsies through the left mid PZ revealed only normal prostatic tissue and areas

of chronic prostatitis. In retrospect, trace $\mathrm{b} 1000 \mathrm{~mm}^{2} / \mathrm{s}$ EPI demonstrates low SI (white arrow in c), which indicates that there is no restricted diffusion in this area. Similarly, there is a benign progressive type I enhancement curve on DCE (d), which further argues against a higher grade tumour. Corresponding histopathology slide (e) demonstrates areas of chronic inflammation 

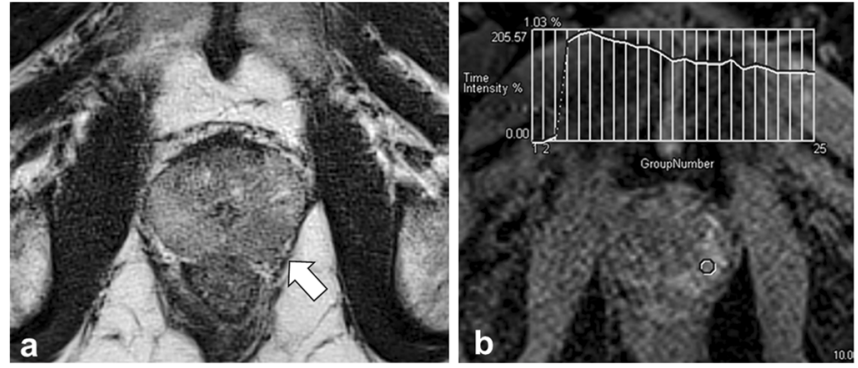

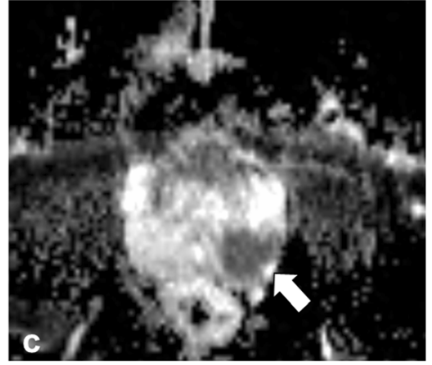

acinar adenocarcinoma $\mathrm{T} 2 \mathrm{~W}$ signal intensity mirrors findings on ADC map, the discordant findings with only minimally decreased T2W signal and profound restricted diffusion can be seen in ductal variant adenocarcinoma. Final histopathology after RP was Gleason score 4+ 4=8 tumour with dominant ductal component

spin-echo techniques [39]. Motion correction with radial acquisition (BLADE, Siemens Healthcare, Malvern, PA, USA; PROPELLER, General Electric Healthcare, Milwaukee, WI, USA) sequences are popular for pelvic imaging because they correct for in-plane rotation and translational artefacts and have been shown to result in improved overall image quality when compared to conventional spin echo [59,60]. A disadvantage of these sequences is decreased image contrast compared to conventional spin echo [59]. Recently, Rosenkrantz et al. demonstrated that a minority of PCa foci may be obscured at T2W MRI when BLADE/PROPELLER is used compared to conventional spin echo [61] (Fig. 12).
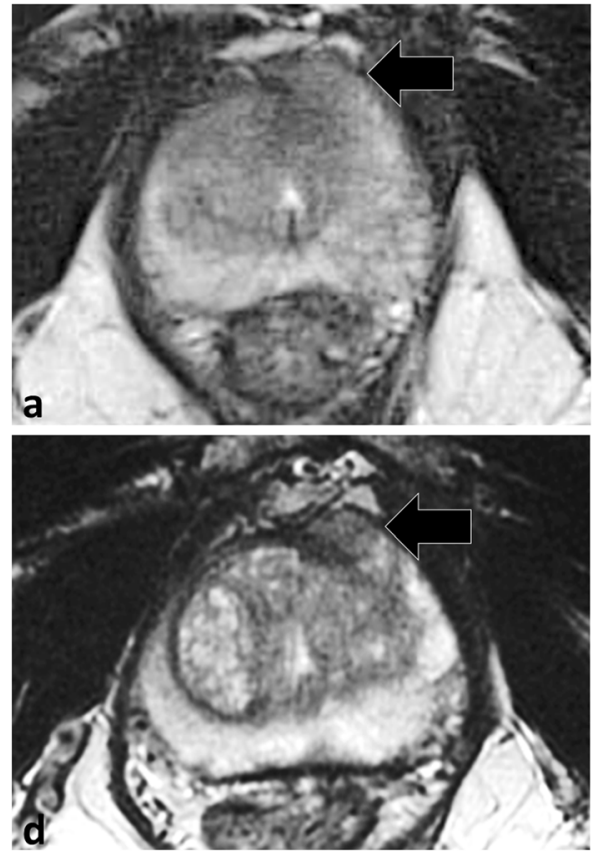

Fig. 12 A 63-year-old patient with elevated PSA and previously negative non-targeted TRUS-guided biopsy with persistent clinical suspicion of prostate cancer underwent MP-MRI in two separate sessions within 3 months demonstrating the loss of contrast with BLADE/ PROPELLER imaging. Axial T2W BLADE $\mathbf{a}, \mathrm{b} 1000 \mathrm{~mm}^{2} / \mathrm{s}$ EPI b and ADC map c demonstrate a suspicious focus of restricted diffusion in the
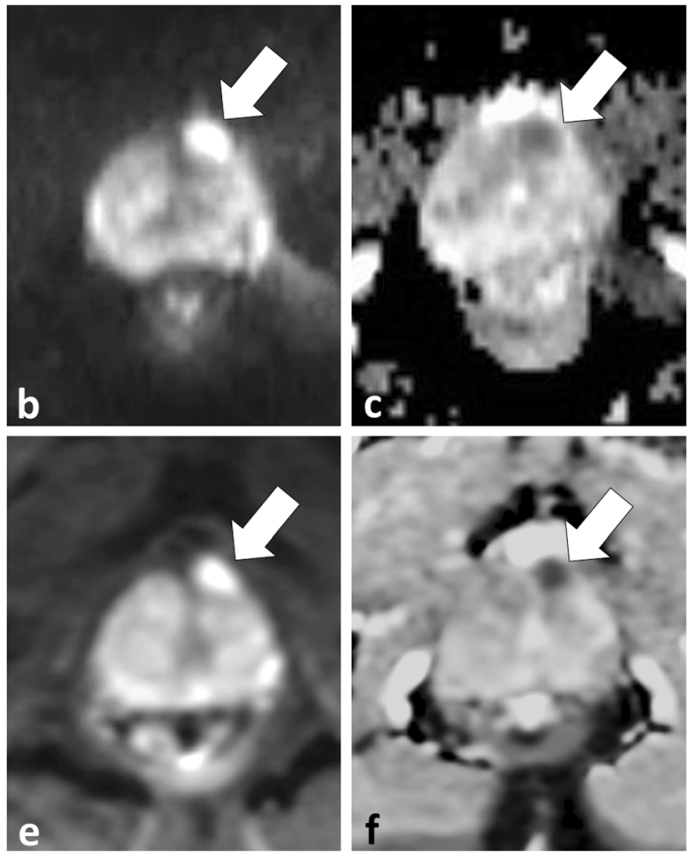

left middle anterior horn of the PZ (white arrows), which is not visible on axial T2W BLADE (black arrow in a). Repeat examination performed using T2W FSE (d) reveals a T2 hypointense nodule in the same location (black arrow in d) with persistent restricted diffusion (white arrows in $\mathbf{e}$ and f) 


\section{(2) Visual/quantitative analysis of DWI for tumour detection/grading is complex}

DWI improves the detection of cancer foci in the PZ and $\mathrm{TZ}$, and ADC values derived from DWI have been repeatedly shown to inversely correlate with Gleason grade of $\mathrm{PCa}[18$, 38]. DWI is the single best imaging test for analysis of the PZ and is used in conjunction with T2W MRI for evaluation of TZ lesions [58, 62]. The visual analysis of DWI must include assessment of trace EPI and should not rely solely upon review of the derived apparent diffusion coefficient (ADC) map. Structures that are inherently of low T2W SI will appear dark on the ADC map because of an inherently low T2W SI (T2 "black-hole" effect) and not from true restricted diffusion (Figs. 1 and 2). Review of trace EPI is critical to avoid this common pitfall because these structures will not appear bright on long ( $\left.\mathrm{b} \geq 1000 \mathrm{~mm}^{2} / \mathrm{s}\right)$ EPI (Figs. 1 and 2). Conversely, PCa (and as discussed some stromal BPH nodules) is of low SI on ADC and will appear bright on the long $\left(b \geq 1000 \mathrm{~mm}^{2} / \mathrm{s}\right)$ EPI (Figs. 3,6 and 7). The use of longer $b$ values $\left(b \geq 1000 \mathrm{~mm}^{2} / \mathrm{s}\right)$ facilitates the detection of cancer foci compared to the benign prostatic tissues [63, 64]. Visual analysis of ADC may be further limited by automatic display settings and can result in the failure to detect $\mathrm{PCa}$ or an underestimation of tumour grade if not displayed properly (Fig. 13). A previous study demonstrated that by using a window width $=1.650$ and lev$\mathrm{el}=1.675 \times 10-6 \mathrm{~mm}^{2} / \mathrm{s}$ for display of ADC, higher grade tumours were more likely to appear dark (Fig. 13) [65].

It has been demonstrated repeatedly that $\mathrm{ADC}$ values inversely correlate to PCa grade [18]. The use of ADC values across MR systems is problematic because of differences related to magnet strength, vendor system and selection of differing b values [57]. Furthermore, a variety of methods to quantify ADC have been described including single region of interest measurement versus whole lesion analysis and mean, median and complex histogram analysis of ADC. Application of quantitative ADC thresholds may be limited by these factors. A recent study demonstrated that the use of an ADC ratio (normalised to adjacent normal PZ) provided a better inter-scanner comparison than the absolute ADC value [57]. Mean ADC values may also be limited due to the variable composition of prostate cancers and when comparing intermediate prostate cancers (Gleason score $3+4=7$ vs. $4+3=7$ ) [66] and studies have demonstrated that the use of the low percentile ADC may be a better reflector of the Gleason score [67, 68]. Regardless of the method used, due to differences across systems, at present, we suggest that the use of quantitative ADC in clinical practice should be applied carefully, derived and validated on an institutional basis.
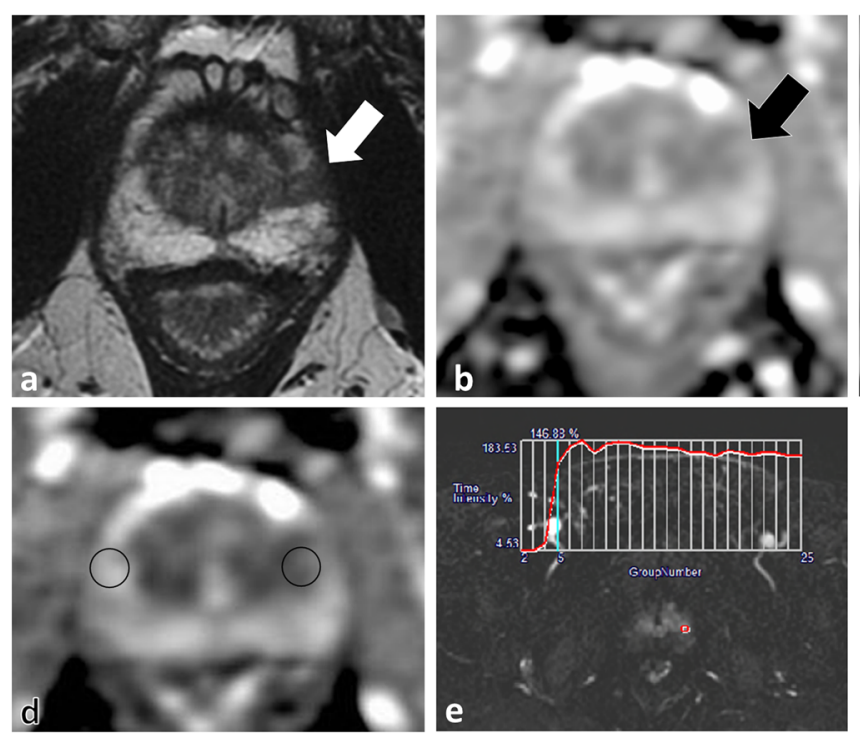

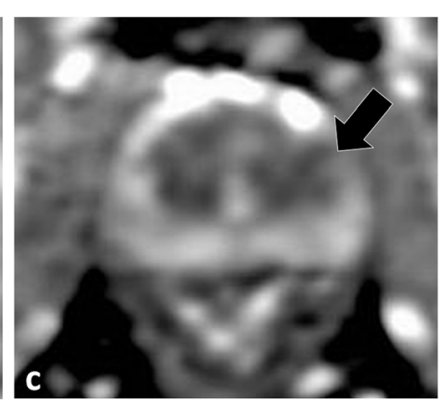

considered to be of low (Gleason score 6) grade using previously reported thresholds. Comparing ADC values across systems is challenging due to a lack of standardization, and an ADC ratio has been previously proposed as a better metric to compare ADC. An ADC value from the contralateral normal PZ obtained at the same level was $2.000 \times$ $10^{-3} \mathrm{~mm}^{2} / \mathrm{s}$, which yields an ADC ratio of 0.73 , which would be compatible with a Gleason score $\geq 7$ tumour based on previously published thresholds. Corresponding DCE image (e) from the same level demonstrated a focal enhancing nodule with a type III contrast curve. Based on the imaging findings, a repeat TRUS-guided biopsy was performed, which demonstrated Gleason pattern 4 in the left apical PZ 

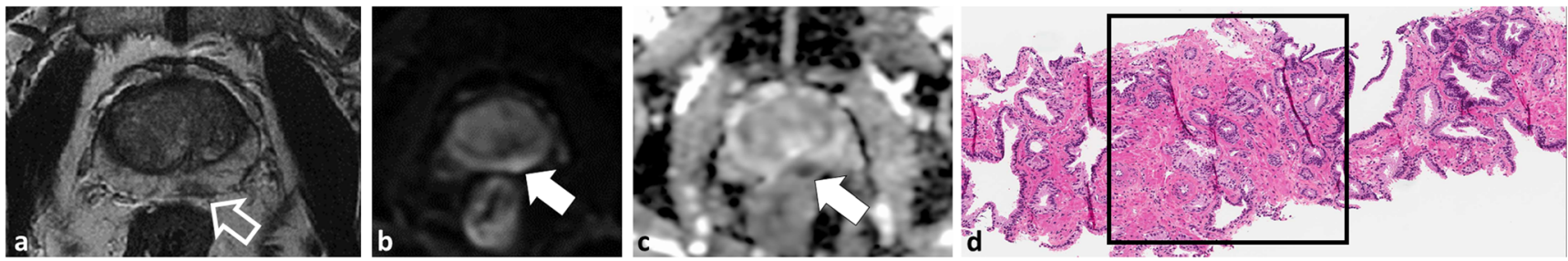

Fig. 14 A 59-year-old patient with low-volume Gleason score $3+3=6$ at non-targeted TRUS biopsy underwent MP-MRI to exclude clinically significant cancer after an increase in PSA. Axial T2W TSE image (a) demonstrates a low T2 SI focus in the left PZ (open white arrow) with restricted diffusion; note increased SI on trace $b 1000 \mathrm{~mm}^{2} / \mathrm{s}$ EPI and low SI on ADC (white arrows in $\mathbf{b}$ and $\mathbf{c}$, respectively). Based on the MRI and clinical findings a diagnosis of potential higher grade tumour was

\section{(3) DCE lacks standardisation and is limited in the TZ}

DCE improves the detection of PCa and DCE parameters correlate with PCa grade [69-71]. Currently, there is no established interpretation criteria for DCE analysis which varies from simple visual analysis to semiquantitative analysis to full quantitative pharmacokinetic modelling [72]. A recent study demonstrated that DCE is underutilised in clinical practice compared to DWI and that semi-quantitative and quantitative analyses were not commonly used [72]. In the revised PIRADS v2.0 guidelines, DCE analysis has been modified from semi-quantitative curve analysis to a simple visual analysis [58]. suggested and a targeted repeat biopsy was performed. At time of repeat biopsy, which used cognitive registration of MP-MRI and TRUS data, no lesion could be identified at TRUS. Only one core biopsy through the left medial mid peripheral zone sextant was performed. Microscopic image from repeat TRUS-guided biopsy (d) demonstrates Gleason pattern 3 tumour. Continued in Fig. 14

Moreover, DCE is very limited for the detection and grading of cancers in the transition zone because of significant overlap with stromal BPH (Fig. 7). Analysis of potential tumour foci in the transition zone should preferentially favour T2W and DWI over DCE. It is currently recognised that the utility of DCE in clinical practice is mainly as a confirmatory sequence for PZ lesions [58]; however, further studies are required to determine the value of DCE in PCa.

\section{(4) Targeted biopsy of MR-detected lesions using TRUS- guidance is challenging}

MP-MRI for PCa has transformed practice, but has created new challenges. Obtaining accurate histological correlation
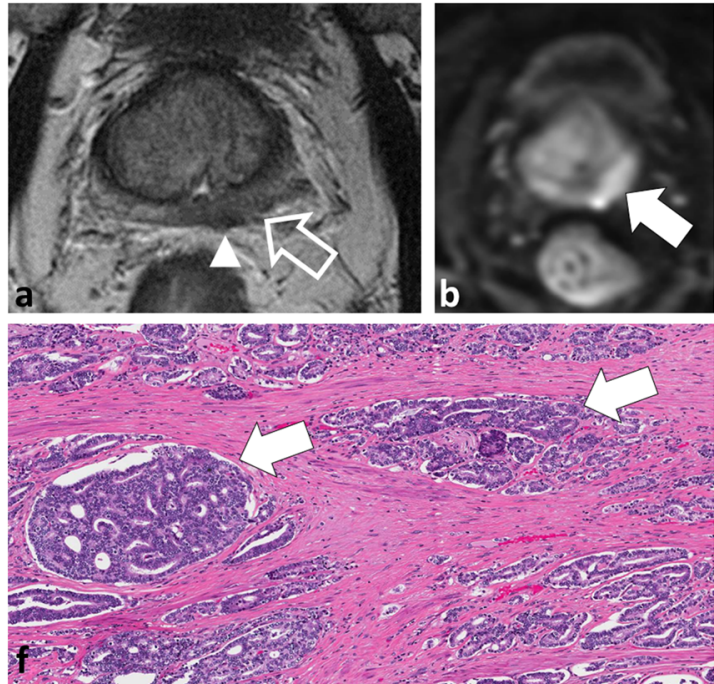

Fig. 15 Although only low-volume Gleason score $3+3=6$ was again noted at repeat TRUS biopsy (Fig. 13), a follow-up MP-MRI was performed 3 months later because of interval doubling of PSA to reevaluate for a focus of higher grade cancer. Axial T2W TSE image (a) demonstrates that the small low T2 SI focus in the left PZ has grown substantially (open white arrow) with bulging and nodular extension into the peri-prostatic fat (arrowhead), which was reported as representing extra-prostatic extension. The lesion again demonstrates restricted diffusion; note increased SI on trace b1000 $\mathrm{mm}^{2} / \mathrm{s}$ EPI and low SI on $\mathrm{ADC}$ (white arrows in $\mathbf{b}$ and $\mathbf{c}$, respectively) and demonstrated an
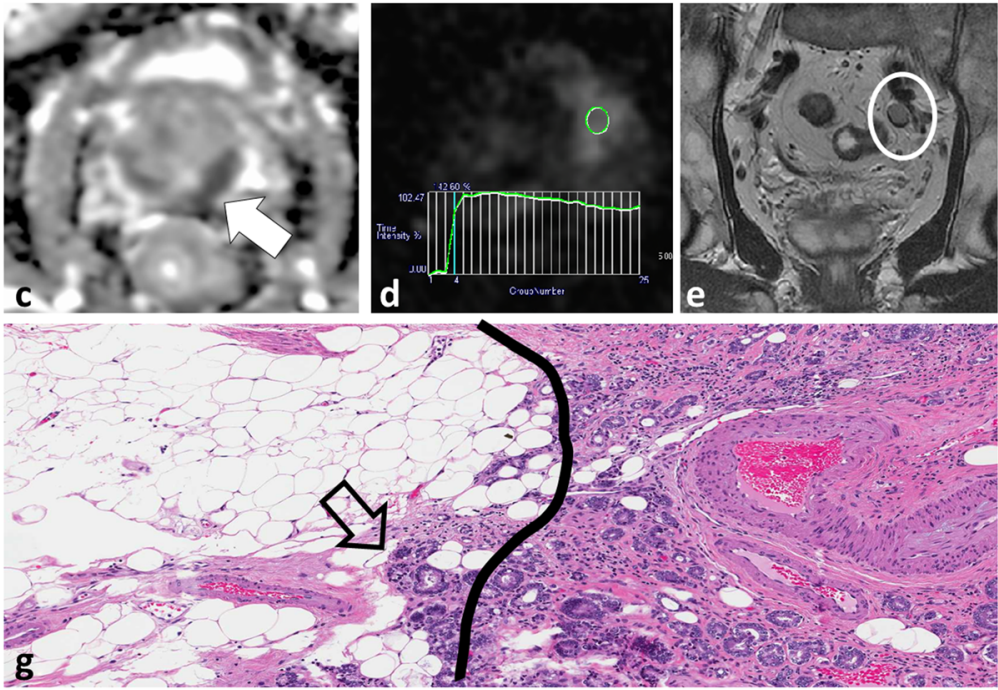

aggressive type III contrast curve on DCE (d). In the interval, a malignant-appearing lymph node developed along the left pelvic sidewall (e). Based on these findings a diagnosis of high-grade tumour with extra-prostatic extension and metastatic adenopathy was provided. The patient underwent RP based on the imaging findings, he declined a repeat biopsy because of previous urosepsis related to prior TRUS biopsy. Corresponding microscopic images (f and $\mathbf{g}$ ) demonstrate Gleason pattern 4 tumour (white arrows in f) and extra-prostatic extension of tumour (open arrow), which is beyond the prostate capsule (black line) and into the peri-prostatic fat $(\mathbf{g})$ 

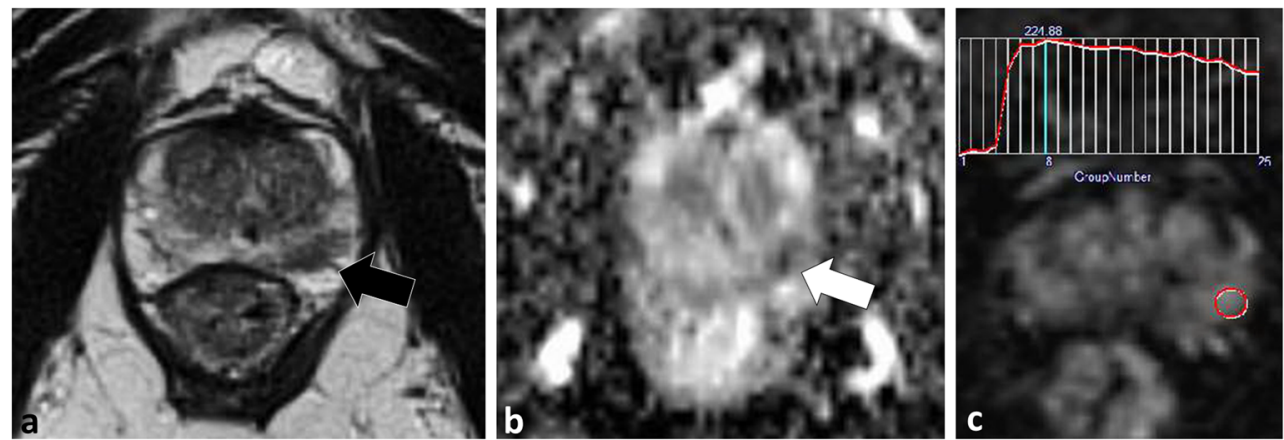

Fig. 16 A 63-year-old patient underwent MP-MRI prior to routine repeat biopsies as part of his active surveillance protocol. Axial T2W TSE (a), axial ADC map (b) and semi-quantitative contrast curve derived from DCE (c) demonstrate a focal low T2W SI lesion (black arrow) with restricted diffusion (white arrow) and type III kinetics in the left middle peripheral zone. At repeat targeted biopsy using cognitive registration, no corresponding lesion could be identified. With a priori knowledge of the location of the lesion at MP-MRI, the TRUS operator performed three core needle biopsies through the left middle lateral and two core needle biopsies through the left middle medial PZ sextants. Results after targeted biopsies were Gleason $4+3=7$ tumour with two out of three core biopsies in the left middle lateral $\mathrm{PZ}$ sextant from lesions detected at MP-MRI may be challenging. MRI guided biopsy is accurate; however, this technique is limited by cost, availability of and access to MRI, prolonged procedure times and patient discomfort [8]. The use of TRUS guidance for biopsy of MP-MRI-detected lesions is currently a preferred option.

Using existing technology, so-called cognitive registration (CR) is performed. CR requires the TRUS operator to mentally integrate MP-MRI with real-time TRUS, identify suspicious MR lesions and, with TRUS guidance, target those lesions for biopsy. Published data regarding the applicability of CR are limited to institutional series and are highly subject to operator experience [8]. TRUS may be limited for the detection of tumours in the PZ and is also limited for assessment of the $\mathrm{TZ}$ and the anterior gland in many patients with enlarged glands due to poor ultrasound beam penetration. A failure to recognise the limitations of TRUS when performing CR biopsies can result in a failure to adequately sample suspicious areas on MP-MRI and ultimately result in delayed diagnosis and therapy for clinically significant $\mathrm{PCa}$ (Figs. 14 and 15). In our experience, cognitively registered TRUS biopsy of MP-MRI-detected lesions can be successful, provided that the TRUS operator recognises the limitations of TRUS for demonstrating a corresponding lesion to that seen on MP-MRI. It is not uncommon for a lesion detected in the PZ or TZ on mp-MRI to be sonographically occult on TRUS and, in these instances, by oversampling the corresponding location using anatomic landmarks, the diagnostic yield can be significantly improved (Fig. 16).

The use of Fusion software (which automatically integrates MP-MRI data with real-time 3D TRUS images) provides an alternative to cognitive registration [8]. Fusion software is not without its own limitations including mainly errors in fusion that relate to the spatial deformation of the prostate at TRUS compared to mp-MRI [8]. Moreover, this technology is expensive and at the moment is available in a few specialised centres, although availability is increasing. Studies comparing cognitively registered targeted TRUS biopsy to software fused targeted TRUS biopsy are lacking and have shown mixed results. While several studies have shown no difference between CR and Fusion software [73, 74], other studies have shown an improvement in the detection rate of cancer using fusion software systems $[75,76]$.

It is critical to emphasise that when a targeted TRUS-guided biopsy performed for a suspicious lesion detected on MP-MRI (using either cognitive registration or fusion software) is negative, the MP-MRI should be reviewed in the context of the biopsy results and other clinical factors in order to consider the possibility of an erroneous targeted biopsy. In these instances, repeat MRI or targeted biopsies can be contemplated.

\section{Conclusion}

In conclusion, MP-MRI has become a critical component for patients being considered for or enrolled in active surveillance protocols for the management of low-grade and low-volume prostate cancers. MP-MRI is a proven imaging modality that can detect clinically significant foci of prostate cancer with high degrees of accuracy; the high negative predictive value of MP-MRI is particularly well suited for the AS of PCa. A number of pitfalls, both interpretive and technical, may be encountered at MP-MRI of the prostate and a failure to recognise these pitfalls in the AS population can result in suboptimal patient care. Targeted biopsies of MP-MR-detected lesions poses a new challenge and opportunity in clinical practice. The limitations of TRUS-guidance for lesion detection during targeted biopsies should be acknowledged in order to improve the diagnostic yield of targeted biopsies. A thorough understanding of these MP-MRI pitfalls is important for the MR practitioner involved in the management of prostate cancer. 
Open Access This article is distributed under the terms of the Creative Commons Attribution 4.0 International License (http:// creativecommons.org/licenses/by/4.0/), which permits unrestricted use, distribution, and reproduction in any medium, provided you give appropriate credit to the original author(s) and the source, provide a link to the Creative Commons license, and indicate if changes were made.

\section{References}

1. National Comprehensive Cancer Network NCCN (2012) Clinical practice guidelines in oncology: prostate cancer. Fort Washington, PA. Available via http://www.nccn.com/files/cancer-guidelines/ prostate/index.html\#/1. Accessed Sept. 132013

2. Prostate Cancer Canada (2014) Prostate cancer Canada network. Toronto, ON Canada. Available via http://prostatecancer.ca. Accessed Sept. 2014

3. European Association of Urology (EAU) (2012) Guidelines on prostate cancer. Arnhem, The Netherlands. Available via http:// www.uroweb.org/gls/pdf $/ 08 \% 20$ Prostate $\% 20$ Cancer_LR $\%$ 20March\%2013th\%202012.pdf

4. Thomsen FB, Brasso K, Klotz LH, Roder MA, Berg KD, Iversen P (2014) Active surveillance for clinically localized prostate cancer-a systematic review. J Surg Oncol 109:830-835

5. Heidenreich A, Bellmunt J, Bolla M et al (2011) EAU guidelines on prostate cancer. Part 1: screening, diagnosis, and treatment of clinically localised disease. Eur Urol 59:61-71

6. Cancer Care Ontario CCO (2012) Prostate cancer treatment pathway. Toronto, ON, Canada. Available via http://www.cancercare. on.ca/common/pages/UserFile.aspx?fileID $=298448$. Accessed Sept. 2014

7. National Institutes of Health NIH (2011) Role of Active surveillance in the management of men with localized prostate cancer. Bethesda, Maryland. Available via http://consensus.nih.gov/2011/ prostate.htm. Accessed Sept. 2014

8. Rothwax JT, George AK, Wood BJ, Pinto PA (2014) Multiparametric MRI in biopsy guidance for prostate cancer: fusion-guided. Biomed Res Int 2014:439171

9. Eskew LA, Bare RL, McCullough DL (1997) Systematic 5 region prostate biopsy is superior to sextant method for diagnosing carcinoma of the prostate. J Urol 157:199-202, discussion 202-193

10. Presti JC Jr, O’Dowd GJ, Miller MC, Mattu R, Veltri RW (2003) Extended peripheral zone biopsy schemes increase cancer detection rates and minimize variance in prostate specific antigen and age related cancer rates: results of a community multi-practice study. $\mathrm{J}$ Urol 169:125-129

11. Babaian RJ, Toi A, Kamoi K et al (2000) A comparative analysis of sextant and an extended 11-core multisite directed biopsy strategy. J Urol 163:152-157

12. Noguchi M, Stamey TA, McNeal JE, Yemoto CM (2001) Relationship between systematic biopsies and histological features of 222 radical prostatectomy specimens: lack of prediction of tumor significance for men with nonpalpable prostate cancer. J Urol 166: 104-109, discussion 109-110

13. Bott SR, Young MP, Kellett MJ, Parkinson MC (2002) Anterior prostate cancer: is it more difficult to diagnose? BJU Int 89:886889

14. Eberhardt SC, Carter S, Casalino DD et al (2013) ACR appropriateness criteria prostate cancer-pretreatment detection, staging, and surveillance. J Am Coll Radiol 10:83-92

15. Barentsz JO, Richenberg J, Clements R et al (2012) ESUR prostate MR guidelines 2012. Eur Radiol 22:746-757
16. Hamoen EH, de Rooij M, Witjes JA, Barentsz JO, Rovers MM (2014) Use of the prostate imaging reporting and data system (PIRADS) for prostate cancer detection with multiparametric magnetic resonance imaging: a diagnostic meta-analysis. Eur Urol 67:11121121

17. de Rooij M, Hamoen EH, Futterer JJ, Barentsz JO, Rovers MM (2014) Accuracy of multiparametric MRI for prostate cancer detection: a meta-analysis. AJR Am J Roentgenol 202:343-351

18. Hegde JV, Mulkern RV, Panych LP et al (2013) Multiparametric MRI of prostate cancer: an update on state-of-the-art techniques and their performance in detecting and localizing prostate cancer. $\mathrm{J}$ Magn Reson Imaging 37:1035-1054

19. Schoots IG, Petrides N, Giganti F et al (2015) Magnetic resonance imaging in active surveillance of prostate cancer: a systematic review. Eur Urol 67:627-636

20. Stamatakis L, Siddiqui MM, Nix JW et al (2013) Accuracy of multiparametric magnetic resonance imaging in confirming eligibility for active surveillance for men with prostate cancer. Cancer 119:3359-3366

21. Da Rosa MR, Milot L, Sugar L et al (2014) A prospective comparison of MRI-US fused targeted biopsy versus systemic ultrasoundguided biopsy for detecting clinically significant prostate cancer in patients on active surveillance. J Magn Reson Imaging 41:220-5

22. National Institute for Health and Care Excellence (NICE). CG175 NG (2014) Prostate cancer: diagnosis and treatment. Available via http://www.nice.org.uk/guidance/cg175. Accessed Dec. 2014

23. McNeal JE (1981) The zonal anatomy of the prostate. Prostate 2:35-49

24. McNeal JE, Redwine EA, Freiha FS, Stamey TA (1988) Zonal distribution of prostatic adenocarcinoma. Correlation with histologic pattern and direction of spread. Am J Surg Pathol 12:897-906

25. Rosenkrantz AB, Taneja SS (2014) Radiologist, be aware: ten pitfalls that confound the interpretation of multiparametric prostate MRI. AJR Am J Roentgenol 202:109-120

26. Vargas HA, Akin O, Franiel T et al (2012) Normal central zone of the prostate and central zone involvement by prostate cancer: clinical and MR imaging implications. Radiology 262:894-902

27. Mai KT, Belanger EC, Al-Maghrabi HM, Robertson S, Wang D, Margnean C (2008) Primary prostatic central zone adenocarcinoma. Pathol Res Pract 204:251-258

28. Cohen RJ, Shannon BA, Phillips M, Moorin RE, Wheeler TM, Garrett KL (2008) Central zone carcinoma of the prostate gland: a distinct tumor type with poor prognostic features. J Urol 179:17621767, discussion 1767

29. Lemaitre L, Puech P, Poncelet E et al (2009) Dynamic contrastenhanced MRI of anterior prostate cancer: morphometric assessment and correlation with radical prostatectomy findings. Eur Radiol 19:470-480

30. Bonekamp D, Jacobs MA, El-Khouli R, Stoianovici D, Macura KJ (2011) Advancements in MR imaging of the prostate: from diagnosis to interventions. Radiographics 31:677-703

31. Akin O, Sala E, Moskowitz CS et al (2006) Transition zone prostate cancers: features, detection, localization, and staging at endorectal MR imaging. Radiology 239:784-792

32. Lawrentschuk N, Haider MA, Daljeet N et al (2010) 'Prostatic evasive anterior tumours': the role of magnetic resonance imaging. BJU Int 105:1231-1236

33. Hoeks CM, Hambrock T, Yakar D et al (2013) Transition zone prostate cancer: detection and localization with 3-T multiparametric MR imaging. Radiology 266:207-217

34. Yoshizako T, Wada A, Hayashi T et al (2008) Usefulness of diffusion-weighted imaging and dynamic contrast-enhanced magnetic resonance imaging in the diagnosis of prostate transition-zone cancer. Acta Radiol 49:1207-1213

35. Oto A, Kayhan A, Jiang Y et al (2010) Prostate cancer: differentiation of central gland cancer from benign prostatic hyperplasia by 
using diffusion-weighted and dynamic contrast-enhanced MR imaging. Radiology 257:715-723

36. Engelbrecht MR, Huisman HJ, Laheij RJ et al (2003) Discrimination of prostate cancer from normal peripheral zone and central gland tissue by using dynamic contrast-enhanced MR imaging. Radiology 229:248-254

37. Turnbull LW, Buckley DL, Turnbull LS, Liney GP, Knowles AJ (1999) Differentiation of prostatic carcinoma and benign prostatic hyperplasia: correlation between dynamic Gd-DTPA-enhanced MR imaging and histopathology. J Magn Reson Imaging 9:311-316

38. Hoeks CM, Barentsz JO, Hambrock T et al (2011) Prostate cancer: multiparametric MR imaging for detection, localization, and staging. Radiology 261:46-66

39. Schieda N, Coffey N, Gulavita P, Al-Dandan O, Shabana W, Flood TA (2014) Prostatic ductal adenocarcinoma: an aggressive tumour variant unrecognized on $\mathrm{T} 2$ weighted magnetic resonance imaging (MRI). Eur Radiol 24:1349-56

40. Claus FG, Hricak H, Hattery RR (2004) Pretreatment evaluation of prostate cancer: role of MR imaging and $1 \mathrm{H}$ MR spectroscopy. Radiographics 24(Suppl 1):S167-180

41. Barrett T, Vargas HA, Akin O, Goldman DA, Hricak H (2012) Value of the hemorrhage exclusion sign on T1-weighted prostate MR images for the detection of prostate cancer. Radiology 263: 751-757

42. Rosenkrantz AB, Kopec M, Kong X et al (2010) Prostate cancer vs. post-biopsy hemorrhage: diagnosis with T2- and diffusionweighted imaging. J Magn Reson Imaging 31:1387-1394

43. Chesnais AL, Niaf E, Bratan F et al (2013) Differentiation of transitional zone prostate cancer from benign hyperplasia nodules: evaluation of discriminant criteria at multiparametric MRI. Clin Radiol 68:e323-330

44. Bouye S, Potiron E, Puech P, Leroy X, Lemaitre L, Villers A (2009) Transition zone and anterior stromal prostate cancers: zone of origin and intraprostatic patterns of spread at histopathology. Prostate 69: 105-113

45. Esen M, Onur MR, Akpolat N, Orhan I, Kocakoc E (2013) Utility of ADC measurement on diffusion-weighted MRI in differentiation of prostate cancer, normal prostate and prostatitis. Quant Imaging Med Surg 3:210-216

46. Nagel KN, Schouten MG, Hambrock T et al (2013) Differentiation of prostatitis and prostate cancer by using diffusion-weighted MR imaging and MR-guided biopsy at $3 \mathrm{~T}$. Radiology 267:164-172

47. Bour L, Schull A, Delongchamps NB et al (2013) Multiparametric MRI features of granulomatous prostatitis and tubercular prostate abscess. Diagn Interv Imaging 94:84-90

48. Shukla-Dave A, Hricak H, Eberhardt SC et al (2004) Chronic prostatitis: MR imaging and $1 \mathrm{H}$ MR spectroscopic imaging findingsinitial observations. Radiology 231:717-724

49. Franiel T, Ludemann L, Rudolph B et al (2008) Evaluation of normal prostate tissue, chronic prostatitis, and prostate cancer by quantitative perfusion analysis using a dynamic contrast-enhanced inversion-prepared dual-contrast gradient echo sequence. Investig Radiol 43:481-487

50. Watanabe Y, Nagayama M, Araki T et al (2013) Targeted biopsy based on ADC map in the detection and localization of prostate cancer: a feasibility study. J Magn Reson Imaging 37:1168-1177

51. Humphrey PA (2012) Histological variants of prostatic carcinoma and their significance. Histopathology 60:59-74

52. Meeks JJ, Zhao LC, Cashy J, Kundu S (2012) Incidence and outcomes of ductal carcinoma of the prostate in the USA: analysis of data from the surveillance, epidemiology, and end results program. BJU Int 109:831-834

53. Seipel AH, Wiklund F, Wiklund NP, Egevad L (2013) Histopathological features of ductal adenocarcinoma of the prostate in 1,051 radical prostatectomy specimens. Virchows Arch 462: $429-436$
54. Amin A, Epstein JI (2011) Pathologic stage of prostatic ductal adenocarcinoma at radical prostatectomy: effect of percentage of the ductal component and associated grade of acinar adenocarcinoma. Am J Surg Pathol 35:615-619

55. Montironi R, Hammond EH, Lin DW et al (2014) Consensus statement with recommendations on active surveillance inclusion criteria and definition of progression in men with localized prostate cancer: the critical role of the pathologist. Virchows Arch 465:623628

56. Amin MB, Lin DW, Gore JL et al (2014) The critical role of the pathologist in determining eligibility for active surveillance as a management option in patients with prostate cancer: consensus statement with recommendations supported by the College of American Pathologists, International Society of Urological Pathology, Association of Directors of Anatomic and Surgical Pathology, the New Zealand Society of Pathologists, and the Prostate Cancer Foundation. Arch Pathol Lab Med 138:1387-1405

57. Coffey N, Schieda N, Cron G, Gulavita P, Mai KT, Flood TA (2014) Multi-parametric (mp) MRI of prostatic ductal adenocarcinoma. J Magn Reson Imaging. doi:10.1002/jmri.24694

58. Weinreb JC, Choyke PL, Cornud F, Haider MA, Katarzyna MJ, Margolis D, Schnall MD, Tempany CM, Thoeny HC, Verma S, Barentsz JO. Prostate imaging and data repording system (PIRADS) version 2.0. Issued jointly by: American College of Radiology (ACR) and European Society of Urogenital Radiology (ESUR). Website. http://acr.org/ /media/ACR/Documents/PDF/ QualitySafety/Resources/PIRADS/PIRADS\%20V2.pdf. Accessed April 2015

59. Froehlich JM, Metens T, Chilla B, Hauser N, Klarhoefer M, KubikHuch RA (2012) Should less motion sensitive T2-weighted BLADE TSE replace cartesian TSE for female pelvic MRI? Insights Imaging 3:611-618

60. Lane BF, Vandermeer FQ, Oz RC, Irwin EW, McMillan AB, Wong-You-Cheong JJ (2011) Comparison of sagittal T2-weighted BLADE and fast spin-echo MRI of the female pelvis for motion artifact and lesion detection. AJR Am J Roentgenol 197:W307-313

61. Rosenkrantz AB, Bennett GL, Doshi A, Deng FM, Babb JS, Taneja SS (2014) T2-weighted imaging of the prostate: impact of the BLADE technique on image quality and tumor assessment. Abdom Imaging 40:552-9

62. Westphalen AC, Rosenkrantz AB (2014) Prostate imaging reporting and data system (PI-RADS): reflections on early experience with a standardized interpretation scheme for multiparametric prostate MRI. AJR Am J Roentgenol 202:121-123

63. Metens T, Miranda D, Absil J, Matos C (2012) What is the optimal $b$ value in diffusion-weighted MR imaging to depict prostate cancer at $3 \mathrm{~T}$ ? Eur Radiol 22:703-709

64. Wang X, Qian Y, Liu B et al (2014) High-b-value diffusionweighted MRI for the detection of prostate cancer at $3 \mathrm{~T}$. Clin Radiol 69:1165-1170

65. Haider MA, van der Kwast TH, Tanguay J et al (2007) Combined T2-weighted and diffusion-weighted MRI for localization of prostate cancer. AJR Am J Roentgenol 189:323-328

66. Rosenkrantz AB, Triolo MJ, Melamed J, Rusinek H, Taneja SS, Deng FM (2015) Whole-lesion apparent diffusion coefficient metrics as a marker of percentage Gleason 4 component within Gleason 7 prostate cancer at radical prostatectomy. J Magn Reson Imaging 41:708-714

67. Lawrence EM, Gallagher FA, Barrett T et al (2014) Preoperative 3 T diffusion-weighted MRI for the qualitative and quantitative assessment of extracapsular extension in patients with intermediate- or high-risk prostate cancer. AJR Am J Roentgenol 203:W280-286

68. Donati OF, Mazaheri Y, Afaq A et al (2014) Prostate cancer aggressiveness: assessment with whole-lesion histogram analysis of the apparent diffusion coefficient. Radiology 271:143-152 
69. Vos EK, Litjens GJ, Kobus T et al (2013) Assessment of prostate cancer aggressiveness using dynamic contrast-enhanced magnetic resonance imaging at $3 \mathrm{~T}$. Eur Urol 64:448-455

70. Isebaert S, De Keyzer F, Haustermans K et al (2012) Evaluation of semi-quantitative dynamic contrast-enhanced MRI parameters for prostate cancer in correlation to whole-mount histopathology. Eur J Radiol 81:e217-222

71. Langer DL, van der Kwast TH, Evans AJ et al (2010) Prostate tissue composition and MR measurements: investigating the relationships between $\mathrm{ADC}, \mathrm{T} 2, \mathrm{~K}($ trans), $\mathrm{v}(\mathrm{e})$, and corresponding histologic features. Radiology 255:485-494

72. Quon JKA, Jain R, Schieda N (2015) Assessing the utilization of functional imaging in multi-parametric $(\mathrm{mp})$ prostate MRI in routine clinical practice. Clin Radiol 70:373-378

73. Wysock JS, Rosenkrantz AB, Huang WC et al (2014) A prospective, blinded comparison of magnetic resonance (MR) imaging- ultrasound fusion and visual estimation in the performance of MR-targeted prostate biopsy: the PROFUS trial. Eur Urol 66: 343-351

74. Puech P, Rouviere O, Renard-Penna R et al (2013) Prostate cancer diagnosis: multiparametric MR-targeted biopsy with cognitive and transrectal US-MR fusion guidance versus systematic biopsy-prospective multicenter study. Radiology 268: 461-469

75. Delongchamps NB, Peyromaure M, Schull A et al (2013) Prebiopsy magnetic resonance imaging and prostate cancer detection: comparison of random and targeted biopsies. J Urol 189:493499

76. Cool DW, Zhang X, Romagnoli C, Izawa JI, Romano WM, Fenster A (2015) Evaluation of MRI-TRUS fusion versus cognitive registration accuracy for MRI-targeted, TRUS-guided prostate biopsy. AJR Am J Roentgenol 204:83-91 